\section{Edited by Kiriakos Xenitidis and Colin Campbell}

\section{Contents}

- Interpretation of ecological data

- Failure to communicate effectively or failure of feedback?

- Cannabis and psychosis

\section{Interpretation of ecological data}

We read the debate on antidepressants and suicide ${ }^{1}$ with interest, especially the issue concerning the importance of controlling for possible confounding variables in ecological studies and the associated difficulties in drawing conclusions from such ecological observations.

One potentially important confounder, which has been overlooked, is the size of the family of origin. Birth cohort studies from Scotland ${ }^{2}$ and Norway ${ }^{3}$ suggest that having elder siblings may be linked with an increased risk of suicide. As the total fertility rates (a reasonable proxy for average family size) fell across most Western countries between the 1950 s and $1970 \mathrm{~s},{ }^{4}$ it is plausible that some of the decline in suicide rates observed from the late 1980s onwards may be, in part at least, a resultant cohort effect.

1 Isacsson G, Rich CL/Jureidini J, Raven M. The increased use of antidepressants has contributed to the worldwide reduction in suicide rates (debate). Br J Psychiatry 2010; 196; 429-33.

2 Riordan DV, Selvaraj S, Stark C, Gilbert JSE. Perinatal circumstances and risk of offspring suicide. Birth cohort study. Br J Psychiatry 2006; 189: 502-7.

3 Gravseth HM, Mehlum L, Bjerkedal T, Kristensen P. Suicide in young Norwegians in a life course perspective: population based cohort study. $J$ Epidemiol Community Health 2010; 64: 407-12.

4 Population Division of the Department of Economic and Social Affairs of the United Nations Secretariat. World Population Prospects: The 2008 Revision Population Database. United Nations, 2009 (http://esa.un.org/unpp).

Daniel Vincent Riordan, West Cork Mental Health Services, Ireland, and Centre for Rural Health Research and Policy, Inverness, UK. Email: vincent.riordan@hse.ie; Cameron Stark, Centre for Rural Health Research and Policy, Inverness, UK

doi: 10.1192/bjp.197.4.332

Author's reply: We welcome Riordan \& Stark's suggestion that declining family size may have contributed to declining suicide rates. I agree. As we noted, there are many potential confounding variables in the relationship between antidepressants and suicide.

Riordan \& Stark cite two birth cohort studies using data linkage that both found that birth order was independently associated with suicide risk. In Riordan et aps 2006 analysis of linked data from the Scottish Morbidity Record and Scottish death records, higher maternal parity, younger maternal age, non-professional parental occupations and low birth weight were all independently associated with higher suicide risk of offspring in young adulthood. ${ }^{1}$ In Gravseth et als study using several Norwegian national registers, suicide risk factors included higher maternal parity, instability of maternal marital status during childhood, low education levels and severe mental illness. ${ }^{2}$ Notably, maternal parity remained a significant risk factor even after adjustment for mental illness.

Data linkage studies such as Riordan et als and Gravseth et als are particularly important in suicide research because of the need for adequately large sample sizes and sufficient statistical power to investigate suicide, a relatively rare event. They are methodologically superior to ecological studies, which are the mainstay of claims that antidepressants reduce suicide. The inherently weak methodology of ecological studies is often compounded by failure to control for potential confounding factors and by biased interpretation.,

Data linkage studies generally reveal multiple significant contributory factors, many of which are linked to social adversity. Many such factors also contribute to other forms of premature mortality such as accidental death and natural death due to preventable conditions. For example, Riordan et al also found an association between higher maternal parity and increased risk of offspring death from causes other than suicide. The aetiological overlap means that primary prevention focusing on shared determinants has greater potential to reduce overall mortality. ${ }^{5}$

Data linkage studies provide valuable evidence that challenges the simplistic belief that depression is the cause of suicide and antidepressants are the solution. As emphasised by De Leo \& Cerin, ${ }^{4}$ suicide is not simply a function of depression, and suicide prevention is far more than a psychiatric enterprise.

\section{Declaration of interest}

I am a member of Healthy Skepticism.

1 Riordan DV, Selvaraj S, Stark C, Gilbert JSE. Perinatal circumstances and risk of offspring suicide. Birth cohort study. Br J Psychiatry 2006; 189: 502-7.

2 Gravseth HM, Mehlum L, Bjerkedal T, Kristensen P. Suicide in young Norwegians in a life course perspective: population-based cohort study. J Epidemiol Community Health 2010; 64: 407-12.

3 Safer DJ, Zito JM. Do antidepressants reduce suicide rates? Public Health 2007; 121: 274-7.

4 De Leo $D$, Cerin, E. More than antidepressants are needed to avert suicide. BMJ 2003; May 15 (http://www.bmj.com/cgi/eletters/326/7397/1008).

5 Neeleman J. A continuum of premature death. Meta-analysis of competing mortality in the psychosocially vulnerable. Int J Epidemiol 2001; 30: 154-62.

Melissa Raven, Department of Public Health, Flinders University, Adelaide South Australia, Australia. Email: melissa.raven@flinders.edu.au

doi: 10.1192/bjp.197.4.332a

\section{Failure to communicate effectively or failure of feedback?}

In reference to a recent study, ${ }^{1}$ we do not agree with the use of email as a sole medium to provide feedback to website administrators. Electronic communication by an unknown, unexpected source has a high chance of ending up in the spam box and going unnoticed by the recipient. Sending the email on a letterhead or with a university logo could not have added enough authenticity to suspicious-looking mail, given that we are all wary of opening emails, let alone attachments, from unknown senders. Further, the recipient may have lacked the expertise to decipher it as genuine feedback. Lack of acknowledgement of the receipt by a large proportion of websites makes us wonder whether the results should be interpreted as a human failure or a technical failure. An alternative medium could have been a fully addressed, official communication posted or couriered personally to the administrator, with a formal acknowledgement of receipt. Another medium could have been follow-up via telephone acknowledging receipt of the email. But an essential component for feedback to be successfully conveyed is to ensure that the message reached the recipient, that the message was at the very least received, if not acknowledged or acted upon. 